\title{
Anatomy-Peritoneal Membrane
}

\section{Can Effluent Matrix Metalloproteinase-2 and Plasminogen Activator Inhibitor-1 be Used as Biomarkers for Fibrosis in Peritoneal Dialysis Patients?}

Background: The peritoneal effluent contains clinical relevant substances derived from intraperitoneal production or transperitoneal transport or both. Matrix metalloproteinase-2 (MMP-2) is a glycoproteinase that cleaves denatured collagen, and complements other collagenases in the degradation of fibrillar collagens. Elevated intraperitoneal levels of plasminogen activator inhibitor-1 (PAl-1) have been demonstrated to be present in patients with intra-abdominal adhesions. Therefore, the aim of this study was to investigate the potential use of MMP-2 and PAl- 1 in effluent as markers in the development of peritoneal alterations.

Methods: For this purpose, the roles of peritoneal transport and local peritoneal production of these parameters was studied. This single-center cohort study included 86 incident PD patients. All patients were treated with biocompatible dialysis solutions and underwent a standard peritoneal permeability analysis (SPA). The presence of local production, as well as correlations between MMP-2, PAl-1 and peritoneal transport parameters, were studied.

Results: Median effluent levels of $21.4 \mathrm{ng} / \mathrm{mL}$ for MMP-2 and $0.9 \mathrm{ng} /$ $\mathrm{mL}$ for PAl-1 were found. Local peritoneal production averaged $90 \%$ of effluent MMP-2 concentration and 74\% for PAl-1. Furthermore, correlations between peritoneal transport parameters and MMP-2 or PAl-1 were present.

Conclusions: This data illustrate the potential of MMP-2 and PAI-1 as biomarkers of peritoneal modifications, but the components of peritoneal transport and local production should be clearly separated in every patient.

Barreto D.L., Coester A.M., Struijk D.G., Krediet R.T. Academic Medical Center, University of Amsterdam, Amsterdam, The Netherlands.

\section{Decorin Gene Therapy via Hybrid Nanoparticles and Adeno-associated Virus Leads to Prevention} of Peritoneal Fibrosis

Background: Peritoneal membrane fibrosis, a complication of long-term peritoneal dialysis (PD) or peritonitis episodes, leads to membrane failure and consequently failure of therapy. Transforming growth factor beta (TGF $\beta$ ) and vascular endothelial growth factor (VEGF) produced by peritoneal mesothelial cells (MCs), induce cell proliferation causing epithelial-mesenchymal transition (EMT) of MCs and lead to peritoneal fibrosis (PF). Decorin, a small leucine-rich proteoglycan and component of extracellular matrix, inactivates all three isoforms of TGF $\beta$, and has been shown to decrease fibrosis in many tissues.

Study design: SD rats divided into 4 groups were studied. First group of animals were naive control, the second group had peritonitis, the third group had peritonitis and decorin gene therapy delivered by Nanoparticles (NP), and the fourth group had peritonitis and decorin gene therapy via adeno-associated virus (AAV). Peritonitis in rats was induced by intraperitoneal injection of Zymosan (a fungal cell wall component). Animals were sacrificed 14 days after the last dose of Zymosan. PET test analyzed functional characteristics of peritoneal membrane. The efficacy of decorin gene therapy was determined by histological Silver stain, Trichome, H\&E staining and TGF $\beta 1$, $\alpha$-smooth muscle actin (SMA), F-actin, Fibronectin, and type I collagen immunostaining. The change in mRNA was quantified with real-time PCR.

Results: All tested parameters show remarkable decrease in PF by decorin therapy delivered via NP or AAV compared to the no therapy groups. Silver staining showed retention of better cell morphology in both AAV and NP groups compared to zymosan group. There was a substantial improvement in the $\mathrm{MC}$ area in animals that received decorin gene therapy compared to untreated group. Marked reduction in fibrosis marker protein such as SMA was detected in abdominal wall, gut, and liver peritoneum in both decorin-treated groups compared to untreated group. H\&E and F-actin staining reaffirmed this observation. The quantitative mRNA measurements showed that decorin gene therapy given via NP or AAV to rats markedly reduced genes causing fibrosis and excessive healing (TGF $\beta$, SMA, and Collagen-I). The NPmediated decorin gene therapy appears to have better efficacy than the AAV-based therapy between the two therapy groups.

Conclusion: Decorin gene therapy is a novel approach to control PF.

Chaudhary K., ${ }^{1,2}$ Tandon A., ${ }^{1,3}$ Moore H., ${ }^{2}$ Khanna R., ${ }^{2}$ Mohan R.R..${ }^{1,3}$ Harry S. Truman VA Hospital, ${ }^{1}$ Columbia, MO; Division of Nephrology, ${ }^{2}$ University of Missouri, Columbia, MO, and ${ }^{3}$ Department of Ophthalmology, University of Missouri, Columbia, MO. 


\section{Anatomy-Peritoneal Membrane}

\section{Influence of Prednisolone on Glucose and Uric Acid Transfer} Across Peritoneal Membrane in Vitro

Objectives: Prednisolone and another glucocorticosteroids are used by selected PD patients because of their underlying diseases, such as peritoneal fibrosis. Although corticosteroids are potent inhibitors of different processes during inflammation, their influence on transport properties of peritoneum is a little known.

Methods: Our study investigated the influence of prednisolone $(0.001 \mathrm{~g} / \mathrm{dL})$ on the glucose $(1.8 \mathrm{~g} / \mathrm{dL})$ and uric acid $(0.02 \mathrm{~g} / \mathrm{dL})$ transfers across isolated parietal peritoneal membrane taken from the anterior abdominal wall of white HYPLUS 59 rabbits and placed inside a modified Ussing-type chamber. Values for transfer from the interstitial (I) to the mesothelial (M) side of membrane $(\mathrm{I}->\mathrm{M})$ and in the opposite direction $(\mathrm{M}->\mathrm{I})$ were calculated using the mathematical model of mass transport and are expressed as a coefficient of diffusive permeability [P (in centimeters per second)]. Four separate series of experiments were done. In the first and second series, we examined glucose transport: 1) in the control conditions (for 120 minutes), and 2) before (15-60 minutes) and after (75-120 minutes) introduction of prednisolone on the $\mathrm{M}$ side of the membrane, respectively. In the third and fourth series, analogical studies were done in the case of uric acid transfers.

Results: In the control series, stability of bidirectional transports for glucose and uric acid were observed. The values of $\mathrm{P} \pm$ standard error of the mean for $\mathrm{I}->\mathrm{M}$ and $\mathrm{M}->\mathrm{I}$ transfers of glucose were respectively $2.489 \pm 0.329$ and $2.259 \pm 0.493$ [all $\times 0.0001 \mathrm{~cm} / \mathrm{s}$ ]. In the case of uric acid $P$ values of transfers were lower and amounted to $1.936 \pm 0.324 \mathrm{~cm} / \mathrm{s}$ and $1.895 \pm 0.596 \mathrm{~cm} / \mathrm{s}$ [all $\times 0.0001 \mathrm{~cm} / \mathrm{s}$ ], respectively. Application of prednisolone diminished glucose bidirectional transfers by at mean $73 \%(p<0.005)$ and transport of uric acid by at mean $19 \%(p<0.004)$.

Conclusion: The obtained results show, that in vitro prednisolone decreased in the greater extent transfer dynamics in the case of glucose than in the case of uric acid. These observations may have clinical importance, especially in patients with disorders of peritoneal permeability, diabetes or hyperuricemia.

Grzelak T., ${ }^{1}$ Czyzewska-Majchrzak L., ${ }^{2}$ Wojciechowska K., ${ }^{1}$ Szary B., ${ }^{1}$ Witmanowski H., ${ }^{2}$ Czyzewska K. ${ }^{1}$ Department of Chemistry and Clinical Biochemistry, ${ }^{1}$ Poznań University of Medical Poznan, Wielkopolska, Poland; Department of Physiology, ${ }^{2}$ Poznań University of Medical Sciences, Poznan, Wielkopolska, Poland.

\section{A Two-hit Approach in the Development of an Experimental Peritoneal Sclerosis Model}

Background: Encapsulating peritoneal sclerosis (EPS) is the most severe complication of long-term peritoneal dialysis in which bowel loops are entrapped in a cocoon of fibrous tissue. Models of EPS are often based on local administration of chemical irritants and, therefore, do not mimic the clinical situation. Our aim was to develop a clinically relevant model with incorporation of renal failure and exposure to conventional dialysis solutions. In the "two-hit" theory on the development of EPS, long-term peritoneal dialysis is considered the first hit. The second hit is the event or series of events that lead to EPS.

Methods: Thirty-six male Wistar rats underwent a peritoneal catheter implantation and a $70 \%$ nephrectomy. They were randomly divided into 3 peritoneal infusion groups. The experimental group was exposed to a $3.86 \%$ glucose-based conventional dialysis solution for 8 weeks, and then received a second hit of $20 \mathrm{~mL}$ intraperitoneal blood administration obtained from donor rats. Two weeks later, the rats were sacrificed. Two control groups were exposed to the conventional dialysis solution alone or to a buffer solution without glucose for 8 weeks. All animals underwent a peritoneal function test at the end of the experiment. The number of peritoneal adhesions was counted at autopsy, and omental tissue was obtained for morphometrics.

Results: The rats that received blood as a second hit had developed numerous intraperitoneal adhesions as seen in EPS, but without cocoon formation. The experimental group had a mean of 4 macroscopic adhesions versus 1 adhesion in both control groups. Microscopically, no differences were present in fibrosis scores and vessel counts between the 3 groups. Also, peritoneal function parameters (MTAC urea, glucose absorption, and ultrafiltration) were similar in all groups.

Discussion:This attempt to develop a new experimental model in which clinically relevant risk factors for EPS are incorporated did not lead to full-blown EPS. Although the severe adhesions that were seen in the experimental "two-hit" group might cause bowel obstruction, no cocoon formation was present. The relatively short infusion period could be the reason that we did not find differences between the groups in microscopy and peritoneal transport.

Conclusion: Modifications of the described rat model are required to develop a clinically relevant EPS model. Besides renal failure and long-term exposure to bioincompatible peritoneal dialysis solutions, a different second hit or several additional hits could be incorporated in an experimental model of EPS.

Vlijm A., ${ }^{1}$ Sampimon D.E., ${ }^{1}$ de Graaff M., ${ }^{1}$ Struijk D.G., ${ }^{1,2}$ Krediet R.T. ${ }^{1}$ Academic Medical Center, ${ }^{1}$ University of Amsterdam, Division of Nephrology, Department of Medicine, Amsterdam, The Netherlands; Dianet Foundation, ${ }^{2}$ Utrecht-Amsterdam, The Netherlands.

\section{Identification of Genes Implicated in Peritoneal}

\section{Membrane Alterations}

Permanent stimulation of peritoneum during peritoneal dialysis (PD) results in increased expression of genes encoding proteins involved in inflammation and tissue remodeling. This leads to release of cytokines, proinflammatory, profibrotic and growth factors, which contribute to peritoneal fibrosis and neoangiogenesis.

Objective: To assess highly expressed genes potentially involved in peritoneal alterations during PD treatment using animal model.

Methods: Thirty-six male Wistar rats after $70 \%$ nephrectomy and with implanted PD catheter were divided into 3 infusion groups. The first group was exposed to a $3.86 \%$ glucose based dialysis solution for 8 weeks and then received a second hit of intraperitoneal blood administration to develop peritoneal sclerosis. Two control groups were exposed either to the glucose based dialysis solutions or to a buffer without glucose for 8 weeks. All 3 groups were sacrificed 2 weeks later. The omental tissue was obtained for the analysis of gene expression using RT-qPCR and morphometrics. Kruskal-Wallis test was used to compare the groups.

Results: Microscopically the groups showed similar fibrosis scores (NS) and very high vessel counts (NS). The experimental group had more intraperitoneal adhesions. In all 3 groups, inflammatory cells and infiltrates were frequently observed. The genes involved in TGFbeta signaling pathway (Bmp1, Fgf1, Bmp6, Tgfb1, Egf), cell proliferation (Csf2, Lif,Inha), angiogenesis (Pgf, Pdgfa, Figf), and inflammation (II4,II3, II2, II18) were more expressed $(p<0,05)$ in the experimental group. No difference was found between control groups (NS).

Conclusion: Already the short-term infusion period, when mild microskopical and no functional changes are observed, provokes activation of cell proliferation, fibrosis, neoangiogenesis, and inflammation genes pathways.

Parikova A., ${ }^{1}$ Vlijm A.., ${ }^{2}$ de Graaf M., ${ }^{2}$ Brabcova I.. ${ }^{3}$ Viklicky O., ${ }^{1}$ Krediet R. ${ }^{2}$ Dept. of Nephrology, ${ }^{1}$ Institute for Clinical and Experimental Medicine, Prague, Czech Republic; Dept. of Nephrology, Aaboratory ${ }^{3}$ Institute for Clinical and Experimental Medicine, Prague, Czech Republic. 